\title{
USO DEL ARTE Y DE LA CREATIVIDAD EN LAS TERAPIAS PSICOSOCIALES. ESTUDIO Y VALORACIÓN DE RESULTADOS DE LA UTILIZACIÓN DE LA FOTOGRAFÍA CON PACIENTES CON UN TRASTORNO GRAVE Y CRÓNICO.
}

\section{Using Art and Creativity in Psychosocial Therapies. Study and Evaluation of results of the use of photography in patients with severe and chronic disorder.}

\author{
Salva Prefasi Gomar, Teresa Magal Royo, Francisco Garde Calvo, \\ José LUIS GIMÉNEZ LÓPEZ \\ Comunicación Audiovisual, Departamento en Tecnologías Gráf cas.
} Escuela Politécnica Superior de Gandia, Universidad Politécnica de Valencia. C/Paranimf, $1 \cdot 46730$ Grao de Gandia (Valencia) Spain. salprego@epsg.upv.es

Recibido: 13 de Junio de 2010

Aprobado: 18 de septiembre de 2010

\begin{abstract}
Resumen:
En este artículo se dará a conocer la experiencia, y posterior investigación, realizada con personas afectadas por una enfermedad mental grave y crónica delTaller de Creatividad y Rehabilitación del Servicio de Salud Mental del Departamento de Salud n ${ }^{\circ} 12$ del Hospital Francisco de Borja de Gandia, España. El curso se centra en formar a pacientes en el uso de la fotografía y de la imagen como medio de expresión y como parte de las terapias psicosociales para mejorar su calidad de vida frente a la sociedad, además de intentar enriquecer su capacidad analítica, no sólo estéticamente respecto a la fotografía, sino también a nivel personal. Aunque existen numerosos estudios científ cos a nivel internacional que avalan el conocimiento y uso de las Terapias Creativas y Artísticas sobre pacientes crónicos, en España este tipo de actuaciones se consideran pioneras, debido a la estructura sanitaria existente, ya que son pocos los centros psiquiátricos nacionales que las desarrollan de forma continuada y con un seguimiento temporal.

Con esta experiencia pretendemos demostramos cómo la fotografía mejora signi f cativamente la calidad de vida de los pacientes crónicos, y no sólo les motiva socialmente para comunicarse a través del ámbito artístico, sino también para utilizar la creatividad como un arma de acción social.
\end{abstract}

Palabras clave: Creatividad, Terapia Psicosocial, Enfermedad Mental

Prefasi, S., Magal, T., Garde, F., Jiménez, J.L. 2011: Uso del Arte y de la Creatividad en las Terapias Psicosociales. Arte, Individuo y Sociedad, 23 (1), 45-54 


\begin{abstract}
Abstrac:
This article will reveal the experiment and the subsequent investigation work carried out with patients having chronic and severe mental illness at the Creativity and Rehabilitation Workshop of the Mental Health Service at the Health Department no. 12 of the Hospital Francisco de Borja of Gandia, SpainThe course focuses on patient training in the use of photography and image as a means of expression and as a part of psychosocial therapies to improve patients' quality of life in front of the society. In addition, it tries to enhance the analytic capacity of the patient not only with regards to the photography aesthetically, but also personally. There are many international scienti $\mathrm{f}$ surveys backing up the knowledge and use of Artistic and Creative Therapy in chronic patients; however, these activities are seen as pioneering actions in Spain given the current health structure, which includes few national psychiatric centers developing them continuously and being temporally monitored.

This experiment demonstrates that photography signif cantly improves the quality of life of chronic patients and motivates them socially to communicate themselves through art, just as it helps them using creativity as a tool for social action.
\end{abstract}

Key words: Creativity, Psychosocial Therapy, Mental Disorders

Sumario: 1.Introducción 2.Objetivos generales de los cursos 3.Metodología del Curso de Fotografía 4. Conclusiones 5.Referencias bibliográf cas.

Este artículo recoge resultados de la investigación para el Trabajo de Tesis Doctoral "La usabilidad y la accesibilidad de herramientas multimedia digitales para personas con una enfermedad mental grave y crónica", f nanciado, en un primer momento, por el Ministerio de Industria,Turismo y Comercio, dentro del Plan Avanza Contenidos con número de expediente: PAV-070000-2007-474, concedido el 19 de diciembre de 2007 y con una duración desde el 1 de junio de 2007 al 31 de marzo de 2009.

\title{
1. Introducción
}

El conocimiento sobre los trastornos mentales severos y persistentes ha tenido que ir abriéndose camino a través de una serie de estigmas y etiquetas que se han ido acuñando a las personas que sufren algún tipo de enfermedad mental (Moreno, 2001). Los médicos y los terapeutas han desarrollado métodos y terapias que proporcionan a este tipo de enfermos un poco de normalidad en sus vidas (McNamee \& Ger gen, 1992). La mayoría de estas terapias para el tratamiento de la esquizofrenia se centran en el ámbito de la biología, la psicología y la creatividad (Richards, 2001). Esta última mediante la utilización de las artes expresivas a través de talleres de música, teatro, dibujo, pintura, etc. (Creek, 2002).

A nivel general, existen numerosas experiencias sobre la creatividad y la expresión artística mediante el dibujo (Oster, Gould Crone \& Gould, 2004) o la pintura, pero, por otro lado, encontramos pocas experiencias de este tipo relacionadas con el ámbito de la fotografía. Este hecho viene determinado, en la mayoría de los casos, por la limitación en conocimientos y manejo de una herramienta, que con el tiempo se ha vuelto cada vez más versátil técnicamente.

La fotografía proporciona la posibilidad de plasmar lo que se está viendo en ese momento, y a través de lo que es capaz de captar el paciente, estimularle para que intente interpretar aquello que le emociona, le motiva y le sorprende.

Desde el año 2000, en el Taller de Creatividad y Rehabilitación del Servicio de Salud Mental del Departamento de Salud no 12 del Hospital Francisco de Borja de 
Gandia, España, se han desarrollado una serie de terapias creativas de carácter grupal enfocadas a acercar diversas disciplinas artísticas a pacientes con un trastorno mental severo de carácter esquizofrénico.

El objetivo de estos talleres es el de facilitar la palabra a las personas que sufren una enfermedad mental y crear espacios de integración real; una integración que implique la construcción de una nueva identidad más allá de la noción de la enfermedad.

El Taller de Creatividad y Rehabilitación es un recurso integrado dentro de la red asistencial de rehabilitación psicosocial de la comarca de La Safor, Valencia. El Grupo de Trabajo de Rehabilitación de la Comisión Sectorial de Salud Mental de la Mancomunitat de Municipis de La Safor ha desarrollado un proceso de rehabilitación que tiene como objetivo garantizar el trabajo coordinado e integrado de todos los recursos y programas rehabilitadores de este área (T aller de Creatividad, CRIS-CD, Viviendas tuteladas, ASAEM, CEEM, etc.).

Desde su creación, el Taller ha organizado 6 cursos de temática diferente, pero con una base artística, según las necesidades de los pacientes: de Música, de Cerámica, de Reciclaje, de Expresión Corporal, de Escritura Creativa y de Fotografía. Hasta hace apenas un año, los talleres se desarrollaban en un aula polivalente de la Casa de Cultura de Rafelcofer cedida por el ayuntamiento para este menesterActualmente, el Taller de Creatividad dispone de un piso como centro neurálgico del grupo, subvencionado en parte por el Ayuntamiento de Gandia.

La duración de los cursos suele variar según el contenido de los mismos. La mayoría se imparte en sesiones de 4 horas a lo largo de una mañana, durante aproximadamente 4 ó 5 meses, dependiendo de la programación de cada uno de ellos y de las actividades propuestas. Hasta la fecha han pasado más de 80 pacientes formalizados en grupos de 10 a 15 personas por curso. En los comienzos del Taller, y para dinamizar al grupo, los cursos tenían una duración media de 5 meses. Hoy por hoy y debido a la magnitud de los proyectos que se desarrollan, los cursos son anuales.

Actualmente, el taller está dirigido por dos profesionales de enfermería (DUE y Auxiliar de psiquiatría) encargadas de elaborar los proyectos y dar continuidad terapéutica a los cursos. Complementariamente, es necesario contar con diferentes profesionales (músicos, artesanos, actrices, artistas plásticos, fotógrafos, profesionales de la comunicación, terapeutas, etc.) con una sensibilidad y aptitud para relacionarse con los pacientes. También, y con cierta regularidad, se debe contar con el apoyo de un psicólogo clínico como punto de ref exión y coherencia sobre el camino que debe seguir cada curso.

Las características de este tipo de talleres creativos pueden resumirse en los siguientes puntos:

- Ser un proyecto concreto y con un resultado objetivo, para que los propios pacientes puedan ver el resultado de su trabajo.

- Poder realizarse en un tiempo específ co y controlado.

- Debe ser reconocido socialmente por el paciente, sus familiares y su entorno. El reconocimiento de su trabajo acerca a los pacientes a la normalidad social. 


\section{Objetivos generales de los cursos}

Los cursos creativos y artísticos desarrollados por el Taller de Creatividad y Rehabilitación persiguen unos objetivos concretos para contrarrestar los síntomas de la enfermedad mental en general, y de la esquizofrenia en particular. A través de estos cursos, se propone hacer del arte y de la creatividad un instrumento de expresión y comunicación entre las personas afectadas por una enfermedad mental grave. Asimismo, se busca la estimulación de estas personas cuando están en momentos de inhibición o desmotivación, para que en un tiempo determinado recuperen el interés por el trabajo, por los estudios y por la vida en general.

La enfermedad mental, en general y la esquizofrenia en particular, se identif can por una serie de manifestaciones que padece el paciente. Uno de los objetivos de estos talleres, es pretender paliar dichos síntomas, que directamente proporcionarán una mejora en la calidad de vida del enfermo. La relación entre los síntomas y estos objetivos se muestra en la Tabla 1. En ella, encontramos que algunos síntomas de la esquizofrenia (Conclusiones obtenidas de la Sesión Clínica del Hospital Francisco de Borja de Gandia de junio, 2006), tratados a través de Terapias Creativas permiten cumplir objetivos concretos que mejoran la calidad de vida del paciente (ver tabla 1).

\begin{tabular}{|l|l|}
\hline \multicolumn{1}{|c|}{ Síntomas de la Esquizofrenia } & \multicolumn{1}{c|}{ Objetivos que se persiguen } \\
\hline Pobreza afectiva. & Restablecimiento de las relaciones personales. \\
\hline Pobreza del lenguaje. & $\begin{array}{l}\text { Verbalización. Hablar de sentimientos y } \\
\text { emociones. }\end{array}$ \\
\hline Abulia $^{\text {a }}$, apatía. $^{\text {Proyecto común motivante. Impulso hacia la }}$ \\
\hline Anhedonia $^{\text {b. }}$ & Búsqueda de lo lúdico, del placer, de la risa, etc. \\
\hline
\end{tabular}

a. Falta de voluntad, o disminución notable de la energía.

b. Incapacidad para experimentar placer, la pérdida de interés o satisfacción en casi todas las actividades.

Tabla 1. Relación entre los síntomas de la esquizofrenia y los resultados que se persiguen con los talleres de creación artística.

Debemos tener en cuenta que este tipo de enfermedades son crónicas. Esta cronicidad implica sobre todo un deterioro cognitivo paulatino, una pérdida de habilidades sociales y un bloqueo mental sobre actividades cotidianas a las que no puede enfrentarse el enfermo, que afectan a su vida social y familiar (Juan J. López-Ibor, Manuel Valdés, 2002). Los talleres de creatividad suponen un trabajo continuo contra los déf cits de la cronicidad de la enfermedad, además de una tarea diaria contra los déf cits sociales que deben superar el paciente y sus familiares. Mediante reuniones grupales (John F. M .Gleeson, Patrick D. McGorry, 2007), los pacientes expresan y comunican sus temores y sus ilusiones, enfrentándose a la enfermedad y a sus síntomas y evitando encerrarse en sí mismos como suele ocurrir en este tipo de enfermos. 
Las siguientes conclusiones fueron obtenidas de la Sesión Clínica del Hospital Francisco de Borja de Gandia de junio de 2006. (Ver tabla 2 y 3).

\begin{tabular}{|l|l|}
\hline \multicolumn{1}{|c|}{ Déficits de la cronicidad (general) } & \multicolumn{1}{c|}{ Resultados obtenidos } \\
\hline Sentimientos de vacío & El tiempo se llena de contenido. \\
\hline Sentimiento de destrucción interna & Metáforas de construcción. \\
\hline Autoimagen de incapacidad & $\begin{array}{l}\text { Expectativas de capacidad, de producción y de } \\
\text { aprendizaje. }\end{array}$ \\
\hline $\begin{array}{l}\text { Sentimientos de impotencia (o en su defensa, } \\
\text { creencias mágicas de omnipotencia) }\end{array}$ & $\begin{array}{l}\text { Apoyar capacidades reales, estimular las partes } \\
\text { sanas. }\end{array}$ \\
\hline Inhibición & Desinhibición. \\
\hline Negación de conf ictos & $\begin{array}{l}\text { Búsqueda de soluciones. Elaboración de } \\
\text { conf ictos. }\end{array}$ \\
\hline Necesidades de satisfacción inmediata & $\begin{array}{l}\text { Demorar la satisfacción. Ayudar a tolerar la } \\
\text { frustración. Trabajar para obtener resultados. }\end{array}$ \\
\hline Atemporalidad (rutinización) & Reintegración en el tiempo. \\
\hline
\end{tabular}

Tabla 2. Relación entre los diferentes déf cits de cronicidad a los que se enfrenta el paciente y lo que se trabaja para superarlos mediante los talleres de creatividad.

\begin{tabular}{|l|l|}
\hline \multicolumn{1}{|c|}{ Déficits sociales } & \multicolumn{1}{c|}{ Resultados obtenidos } \\
\hline Aislamiento social & Formación y pertenencia a un grupo. \\
\hline Ruptura biográf ca & Historia en el grupo. \\
\hline Marginalidad, desvinculación & $\begin{array}{l}\text { Integración y participación en los recursos } \\
\text { comunitarios. }\end{array}$ \\
\hline Incapacitación laboral & Sentimiento de utilidad y normalidad. \\
\hline
\end{tabular}

Tabla 3. Relación entre los diferentes déf cit sociales y los resultados obtenidos

\subsection{Objetivos específicos del curso de fotografía}

El objetivo principal del curso de fotografía es el de mejorar la calidad de vida de los participantes del Taller. Como objetivos secundarios pretendemos que los participantes se diviertan, jueguen con la cámara, aprendan a ver el mundo de otra manera, que se atrevan a contemplar las pequeñas cosas que les rodea, y por supuesto, dar a conocer su trabajo a la sociedad y que sea reconocido su esfuerzo mediante una exposición fotográf ca con las imágenes captadas por ellos mismos.

Con el curso de fotografía los alumnos aprendieron a enfrentarse a los déf cits de la cronicidad de una forma más concreta y particular. (Ver Tabla 4). 


\begin{tabular}{|l|l|}
\hline \multicolumn{1}{|c|}{ Déficits de la cronicidad } & \multicolumn{1}{c|}{\begin{tabular}{c}
\multicolumn{1}{c|}{ Resultados obtenidos con el curso de } \\
fotografia
\end{tabular}} \\
\hline Sentimientos de vacío & $\begin{array}{l}\text { El tiempo se llena de contenido: No sólo aprendo } \\
\text { cosas sobre la fotografía sino también aprendo } \\
\text { a hacer fotos mediante las sesiones prácticas del } \\
\text { curso. }\end{array}$ \\
\hline Sentimiento de responsabilidad & $\begin{array}{l}\text { Responsabilidad con el material fotográf co y la } \\
\text { propia cámara. }\end{array}$ \\
\hline Sentimiento de respeto & $\begin{array}{l}\text { Respeto hacia mis compañeros y hacia las } \\
\text { personas y las cosas que quiero fotograf ar. }\end{array}$ \\
\hline Autoimagen de incapacidad & $\begin{array}{l}\text { Expectativas de capacidad, de producción } \\
\text { artística de calidad. Capacidad para realizar una } \\
\text { exposición fotográf ca. }\end{array}$ \\
\hline Inhibición & $\begin{array}{l}\text { Desinhibición artística. Me atrevo a preguntar, } \\
\text { a opinar. Hago las fotos que quiero. Me atrevo a } \\
\text { posar. }\end{array}$ \\
\hline Incapacitación laboral & $\begin{array}{l}\text { Trabajar y esperar resultados. La ilusión de ver } \\
\text { las fotos que se hacen, de una semana a otra. } \\
\text { La satisfacción de elegir las mejores para la } \\
\text { exposición, y saber porqué son las mejores. }\end{array}$ \\
\hline Necesidades de satisfacción inmediata & $\begin{array}{l}\text { Formación y pertenencia a un grupo de } \\
\text { fotografía con las mismas inquietudes y metas } \\
\text { que yo: organizar una exposición fotográf ca. }\end{array}$ \\
\hline Aislamiento social & $\begin{array}{l}\text { Integración y participación, cada vez mayor, a } \\
\text { medida que el curso va llegando a su f n, y la } \\
\text { f nalidad del mismo se ve cada vez más cerca. }\end{array}$ \\
\hline Marginalidad, desvinculación & $\begin{array}{l}\text { Sentimiento de utilidad, de normalidad y } \\
\text { productividad sin estar limitados a la mera } \\
\text { enfermedad. }\end{array}$ \\
\hline inquietudes & $\begin{array}{l}\text { El curso plantea preguntas pero no da las } \\
\text { respuestas. Vuelven a aparecer inquietudes en } \\
\text { mi vida. }\end{array}$ \\
\hline a
\end{tabular}

Tabla 4. Relación entre los diferentes déf cits de cronicidad a los que se enfrenta el paciente y lo que se trabaja para superarlos mediante el curso de fotografía.

\section{Metodología del curso de fotografía}

El curso de fotografía está enfocado a pacientes crónicos de esquizofrenia con sintomatología residual y cuya actividad está reducida a la estricta supervivencia (pasear, ver la TV, etc.). Este grupo de pacientes reside en poblaciones periféricas de Gandia en un radio de $15 \mathrm{Km}$. La asistencia es voluntaria, con una presencia completa al curso del $95 \%$ de los pacientes.

El curso de fotografía se inició en el año 2005, y en el momento de su impartición el grupo estaba formado por cuatro hombres y seis mujeres de edades comprendidas 
entre los 18 y los 65 años. Todos ellos padecían un trastorno mental severo y persistente, y en el momento de la impartición del curso, se encontraban en una situación estable de su enfermedad.

En los siguientes años el número de alumnos ha ido aumentando llegando a un máximo de 18, 15 de los cuales se mantienen en la actualidad. El índice de bajas, a lo largo de los tres primeros años del curso, se encuentra entre un 5\% y un $10 \%$, dependiendo de las circunstancias personales de los participantes. En nuestro caso en particular hemos tenido dos bajas: una, por motivos de trabajo y otra porque no le gustaba la fotografía.

Durante el primer año del curso de fotografía, se busca utilizar la creatividad y el arte como una forma de terapia. Se trata de utilizar la creatividad como una flosofía y como un recurso que enfoca y potencia las partes más sanas de las personas, posibilitando derivar ciertas energías internas hacia el exterior; utilizar la creatividad que invita a la ruptura de límites como un acto de valentía, que estimula a experimentar, a investigar, a arriesgarse, a mirar y a ser mirados, a ser escuchados, invita a jugar...

Los contenidos formativos del curso de fotografía están divididos en tres partes. Una parte teórica, una parte práctica y el desarrollo de un proyecto fotográ f co. A lo largo de los tres cursos de fotografía los proyectos han ido variando desde una exposición fotográf ca, con el material obtenido por los alumnos durante el curso (I tú què mires?), hasta la publicación de un libro de imágenes y textos creativos (Sentiments en (des)equilibri) o la edición de agendas anuales con temáticas diversas.

La parte teórica se centra en dar a conocer la Historia de la Fotografía mediante las propias imágenes que la componen, de modo que el alumno vaya adquiriendo una cultura visual. Debido a la atención limitada que sufren este tipo de pacientes, se debe tener muy presente el tiempo que se dedica a cada sesión teórica. En nuestro caso en particular, este tiempo no supera los 45 minutos. Por lo tanto, y para desarrollar esta parte, presentamos una serie de imágenes, desde los inicios de la fotografía hasta la actualidad, en forma de pase de diapositivas. Mediante preguntas y explicaciones buscamos el diálogo y la participación de los pacientes que, en cualquier momento, pueden opinar sobre una imagen, un autor, etc.

Para mostrar una evolución coherente, dividimos las imágenes en tres grupos temporales: inicios de la fotografía, que recogía desde las primeras imágenes obtenidas por Niépce hasta f nales del siglo XIX, imágenes del siglo XX y la fotografía en la actualidad dividida en cuatro grupos: fotoperiodismo, fotografía de moda, de arquitectura y fotografía de bodegón.

Pensamos y creemos, y la práctica así nos lo ha con $\mathrm{f}$ rmado, que es positivo para los alumnos que vean cuantas más imágenes mejor y que puedan disponer de una visión general para poder tener referentes visuales a la hora de captar sus propias imágenes. De esta manera, se evita caer en las convenciones o clichés fotográ f cos más frecuentes. Por ejemplo, habitualmente el retrato de una persona se ciñe solamente al rostro de la persona. En el curso, intentábamos eliminar estas limitaciones a través de fotógrafos para los cuales el retrato es, además, las manos, los ojos, los pies, el cuello, etc.

A nivel práctico, se centra en el manejo de la cámara a niveles básicos de funcionamiento y en el conocimiento esencial de las técnicas de revelado. Por operatividad, 
en la mayor parte de las ocasiones utilizamos cámaras de usar y tirar o cámaras compactas "de ir por casa" que el alumno aporta a nivel personal.

Durante la parte práctica se realizan salidas con los alumnos para la toma fotográf ca. Inicialmente, son salidas cortas para completar las sesiones teóricas, pero a medida que va avanzando el curso, se preparan salidas con temas fotográ $f$ cos específ cos.

Finalmente y para completar la fase práctica del curso, se realiza, anualmente, un proyecto fotográf co. En este primer curso de fotografía la f nalidad ha sido una exposición fotográf ca donde los alumnos muestran sus mejores trabajos. Es una exposición hecha y or ganizada por los propios participantes del taller, por lo tanto, el hecho de contar con ellos hace que algo que en un principio ven imposible, como la organización y montaje de una exposición, se convierta en algo cada vez más palpable, más real, haciendo crecer sus expectativas e ilusiones semana a semana.

Para la selección de las imágenes se colocan todas las fotos ordenadas sobre una mesa y se abre un debate sobre qué fotos elegir y por qué o qué fotos desechar y por qué. De todas las imágenes preseleccionadas, se hace un análisis visual para proporcionar a los alumnos una base estética a la hora de futuras tomas fotográ f cas. Este proceso es muy importante, no sólo para la exposición, pues de él van sur giendo las fotografías f nales, también es importante para los pacientes. El hecho de verse involucrados en el proceso de selección y posteriormente, en el de enmarcado y montaje de la exposición, hace que los pacientes vean materializarse un proyecto del que ellos son los protagonistas.

\section{Conclusiones}

La creatividad y las distintas artes expresivas puede resultar una magní f ca terapia rehabilitadora para aquellas personas que sufren un trastorno mental severo y persistente como la esquizofrenia (Atkinson y Wells, 2000).

De todos los cursos or ganizados por el Taller de Creatividad y Rehabilitación, el de Fotografía es el que más interés despierta en la mayoría de los pacientes. $\mathrm{Su}$ impacto en los participantes es evidente en el momento en el que todos se atreven, con toda naturalidad, a manejar una cámara fotográ f ca. Además, ver los resultados que ellos mismos obtienen, se convierte en un estímulo para querer tener su propia cámara. En más de un caso, después del curso de fotografía, los pacientes continúan llevando su cámara encima, encuadrando, fotogra fando y manifestando su visión creativa. El hecho de haber participado, les hace ver las cosas de una forma diferente.

Debido al éxito obtenido durante los tres cursos realizados podemos decir que la experiencia nos ha servido para evaluar el impacto que ha tenido en sus vidas los cursos en general, y la fotografía en particular, y de qué manera ha servido para desarrollar más sus habilidades sociales, evitar recaídas y mejorar su calidad de vida. A continuación, enumeramos una serie de objetivos generalizados en la mayoría de los integrantes del grupo, que hemos observado y analizado después de cada curso:

- En general, podemos decir que los pacientes que participan en los cursos no tienen recaídas en la enfermedad de carácter signif cativo.

- Aporta una mayor integración social de los participantes. 
- Reanudación de actividades laborales y académicas que estaban abandonadas debido a la enfermedad.

- El curso de fotografía les da la energía necesaria para crear una asociación "Col. lectiu Obertament" (una de las pocas asociaciones, si no la única, en España compuesta únicamente por personas afectadas por una enfermedad mental grave), que utiliza la creatividad como una forma de expresión, comunicación y acción social.

- Poco a poco, los propios pacientes verbalizan su satisfacción personal y grupal.

- Para ellos, el hecho de mirar el mundo a través de un objetivo, se va convirtiendo en algo natural. Van cogiendo el hábito de mirar, observar y ver.

- Capacidad para utilizar las cámaras, capacidad para compartir el carrete, para comunicarse entre ellos, capacidad para elegir las fotografías, capacidad para mantener la ilusión hasta el día de la inauguración de la exposición.

- Mejoran sus relaciones sociales y familiares.

- Se sienten valorados y crece la autoestima al estar haciendo una obra socialmente valorada.

Además, los alumnos aprenden a mirar hacia fuera, acostumbrados como están a mirar hacia su interior, hacia ellos mismos y hacia el caos que les rodea. Esta nueva experiencia les enseña a colocar la mirada en el exterior , a reconocer la belleza de las cosas que existen a su alrededor, a contemplar la vida. Además, les proporciona una motivación importante para poder enfrentarse a cualquier tipo de problema, un elemento decisivo, más allá de las habilidades sociales, que este tipo de pacientes necesita para sentirse más cerca de su normalidad. El curso de fotografía, más allá de la mera f nalidad de una primera exposición, les aporta algo mucho más trascendente para ellos, algo que hasta entonces no se han cuestionado; plantea preguntas pero no da las respuestas, les hace despertar inquietudes que la propia enfermedad ha borrado de sus vidas.

\section{Bibliografía}

Atkinson, K. y Wells, C.; 2000: Creative Therapies: A Psychodynamic Approach Within Occupational Therapy. Nelson Thornes. 159-168.

Creek, J.; 2002: Occupational Therapy and Mental Health: Principles, Skills and Practice. Section 4: Creative Activities. Elsevier Health Sciences. 265-274.

Creek, J; 2002: Occupational Therapy and Mental Health: Principles, Skills and Practice. Section 5: Long-term illness. Elsevier Health Sciences. 335-352.

Conclusiones obtenidas de la Sesión Clínica del Hospital Francisco de Borja de Gandia de junio, 2006.

Conclusiones obtenidas de la Sesión Clínica del Hospital Francisco de Borja de Gandia de junio, 2006.

Gleeson, F. y McGorry , P.D.; 2007: Intervenciones Psicológicas en la Psicosis Temprana. Desclee. 187 y sig.

López-Ibor, J.J. y Valdés, M.; 2002: DSM-IV-TR. Criterios Diagnósticos. Breviario. Elsevier Masson. 143-145. 
McNamee, S. y Gergen, K.; 1992:Therapy as Social Construction. Sage Publications Ltd.

Moreno Cidoncha, E.; 2001: Esquizofrenia. Psicología On line. s

Oster G.D., Gould Crone, P. y Gould, P.; 2004: Using Drawings in Assessment and Therapy: A Guide for Mental Health. Psychology Press. 38-39.

Richards, R.; 2001: Creativity and the Schizophrenia Spectrum: More and More Interesting. Creativity Research Journal, Volume 13, Issue 1. 111 - 132.

Sass, L.A. y Schuldberg, D.; 2001: Introduction to the Special Issue: Creativity and the Schizophrenia Spectrum. Creativity Research Journal, Volume 13, Issue 1 , pages $1-4$. 